\title{
BRUNO DAUCÉ
}

Univ. Angers, GRANEM, SFR Confluences, F-49000 Angers, France

\section{Corps des professeurs des universités en sciences de gestion et du management}

\section{La menace est ailleurs!}

\begin{abstract}
A ujourd'hui, ce n'est plus la recherche qui alimente les débats de bon nombre d'enseignants-chercheurs, mais les projets de réforme qui concernent le monde universitaire. Après les débats sur la LPR (loi de programmation de la recherche 2021-2030) (Musselin, 2020 ; Stiegler et Pébarthe, 2020), la discipline des sciences de gestion s'anime en raison de la modification des conditions de recrutement des Professeurs des Universités (PU) par le ministère de tutelle. Les réseaux sociaux s'agitent et circule sous le manteau un point de vue à paraître dans la Revue française de gestion. Cet article de Paché (2021) s'intitule : "Menaces sur le corps des professeurs des universités en sciences de gestion et du management ? » (Paché, 2021).

Le titre des cet article pose donc la question des menaces qui pèseraient sur le corps des PU. Cependant, la question est vite écartée pour affirmer que : «Ce point de vue, au ton
\end{abstract}


volontairement iconoclaste, souhaite ainsi indiquer que des menaces planent sur le corps des PU 06 (sciences de gestion), avec les conséquences dramatiques que l'on peut imaginer, et qu'il s'avère urgent d'en prendre conscience dès maintenant. » Il n'y a donc plus de question mais plutôt une certitude, la situation est urgente et bientôt dramatique : le corps des PU est menacé ! Il nous semble important de pouvoir débattre de cette affirmation. Nous nous appuierons pour cela sur les données mises à disposition par le ministère l'Enseignement supérieur et de la Recherche ${ }^{1}$. Le corps des PU est-il réellement menacé ? Si oui, d'où vient la menace?

\section{I - COMMENT DEVIENT-ON PU ?}

Dans les disciplines juridique, politique, économique ou de gestion il existe deux voies de recrutement pour devenir Professeur des Universités (PU) qui sont régies par le décret $n^{\circ} 84-431$ du 6 juin 1984 :

- les concours nationaux d'agrégation sur épreuves destinés aux candidats titulaires d'un doctorat ou d'un diplôme équivalent (article 49-2) ;

- les concours sur emplois organisés dans les conditions prévues pour les articles 46-1 46-2 46-3 46-4 46-5.

Désormais, les concours sur emplois sont organisés de la manière suivante :

- 46-1 (dite voie normale) (réservée aux MCF HDR) ;

- 46-2 (réservée aux MCF sous conditions d'ancienneté et de mobilité) ;

- 46-3 (dite voie longue) (réservée aux MCF HDR, sous conditions d'ancienneté) ;
- 46-4 (dite voie professionnelle) (sous conditions d'ancienneté et d'activités) ;

- 46-5 (réservée aux MCF ayant exercé des responsabilités importantes).

Pour la plupart des maîtres de conférences, les possibilités de devenir Professeur des Universités (PU) se réduisent à : l'agrégation, la voie normale (46-1) et la voie longue (46-3). La voie 46-2 semble elle très spécifique et nous n'avons pas identifié de recrutements qui aient eu lieu dans ce cadre. Les deux autres voies concernent également peu de postes et des profils bien particuliers (tableau 1).

C'est le décret n ${ }^{\circ} 2014-997$ du 2 septembre 2014 qui a introduit la possibilité pour les disciplines juridiques (section 01 à $03 \mathrm{du}$ $\mathrm{CNU}$ ), science politique (04), sciences économiques (05) et sciences de gestion (06) de pouvoir recruter leurs PU comme les autres sections CNU, par la voie normale (article 46-1), et pas seulement par la voie de l'agrégation ou de la mutation.

Ce décret a également introduit la possibilité de déroger au contingentement qui prévoyait que l'agrégation devait représenter au moins la moitié du nombre total de postes de professeurs proposés. Les disciplines sciences économiques (05) et sciences de gestion (06) ont ainsi expérimenté le décontingentement depuis 2015. Cette expérimentation a pris fin en décembre 2020 après avoir donné lieu à une évaluation par le Hcéres (Haut Conseil de l'évaluation de la recherche et de l'enseignement supérieur) (Cosnard et Dubois, 2019 ; Cosnard et Cartapanis, 2019).

En février 2021, la LPR (loi de programmation de la recherche 2021-2030) a supprimé

1. L'auteur remercie Jérôme Tourbeaux pour son aide dans l'identification et la compréhension des différents jeux de données disponibles. 
Tableau 1 - Recrutements PU de 2015 à 2019 en section 06

\begin{tabular}{|l|c|c|c|c|c|c|}
\hline Recrutements PU section CNU 06 & $\mathbf{2 0 1 5}$ & $\mathbf{2 0 1 6}$ & $\mathbf{2 0 1 7}$ & $\mathbf{2 0 1 8}$ & $\mathbf{2 0 1 9}$ & Total \\
\hline $46-1$ & 11 & 34 & 34 & 26 & 26 & 131 \\
\hline $46-3$ & 5 & 4 & 1 & & & 10 \\
\hline $46-4$ & & & & & & 0 \\
\hline $46-5$ & & 1 & & & & 1 \\
\hline Mutation / détachement & 12 & 13 & 5 & 2 & 1 & 33 \\
\hline Agrégation externe & 17 & & 7 & & 4 & 28 \\
\hline Total & $\mathbf{4 5}$ & $\mathbf{5 2}$ & $\mathbf{4 7}$ & $\mathbf{2 8}$ & $\mathbf{3 1}$ & $\mathbf{2 0 3}$ \\
\hline
\end{tabular}

l'obligation d'être qualifié par le CNU aux fonctions de professeur des universités pour tous candidats qui ont la qualité de $\mathrm{MCF}$ titulaire. La LPR introduit également la possibilité de recruter sur un contrat à durée déterminée un docteur pour une durée minimale de 3 à 6 ans sur une chaire de professeur junior. À l'issue de son contrat, le professeur junior pourra être titularisé dans le corps des professeurs d'université.

\section{II - LE CORPS DES PU EST-IL MENACÉ ?}

Pour tenter de répondre à cette question faisons un point sur la situation des PU au sein de la section CNU 06. À cette fin, nous utilisons différents jeux de données disponibles sur le site internet du ministère de l'Enseignement supérieur, de la Recherche et de l'Innovation ainsi que sur la plateforme « open data » de ce ministère. Le premier jeu de données concerne les effectifs d'enseignants-chercheurs et d'enseignants titulaires en activité, affectés dans les établissements publics sous tutelle du ministère en charge de l'Enseignement supérieur et de la Recherche de 2010-2011 à 2019-2020 ${ }^{2}$. Les autres jeux de données correspondent aux tableaux associés aux notes de la DGRH (Direction générale des ressources humaines) consacrées à la trajectoire professionnelle des enseignants-chercheurs pour les campagnes 2015 à 2019.

En 2019-2020, le corps des PU représente dans notre section $23,99 \%$ des enseignantschercheurs (1629 MCF et 514 PR en section CNU 06). Comparée aux autres sections, la part des PU apparaît faible. En effet, nous restons loin des sections qui nous sont proches et appartiennent à la grande discipline « Droit» (figure 1).

Nous sommes également loin si l'on prend en compte l'ensemble des sections des grandes disciplines 1 à 4 (droit, lettres, sciences,

2. Les enseignants titulaires dans les établissements publics de l'enseignement supérieur. (2020, octobre 28). https:// mesr.opendatasoft.com/explore/dataset/fr-esr-enseignants-titulaires-esr-public/information/?disjunctive.annee 
Figure 1 - Part des PU au sein des enseignants-chercheurs (2019-2020) (grande discipline droit)

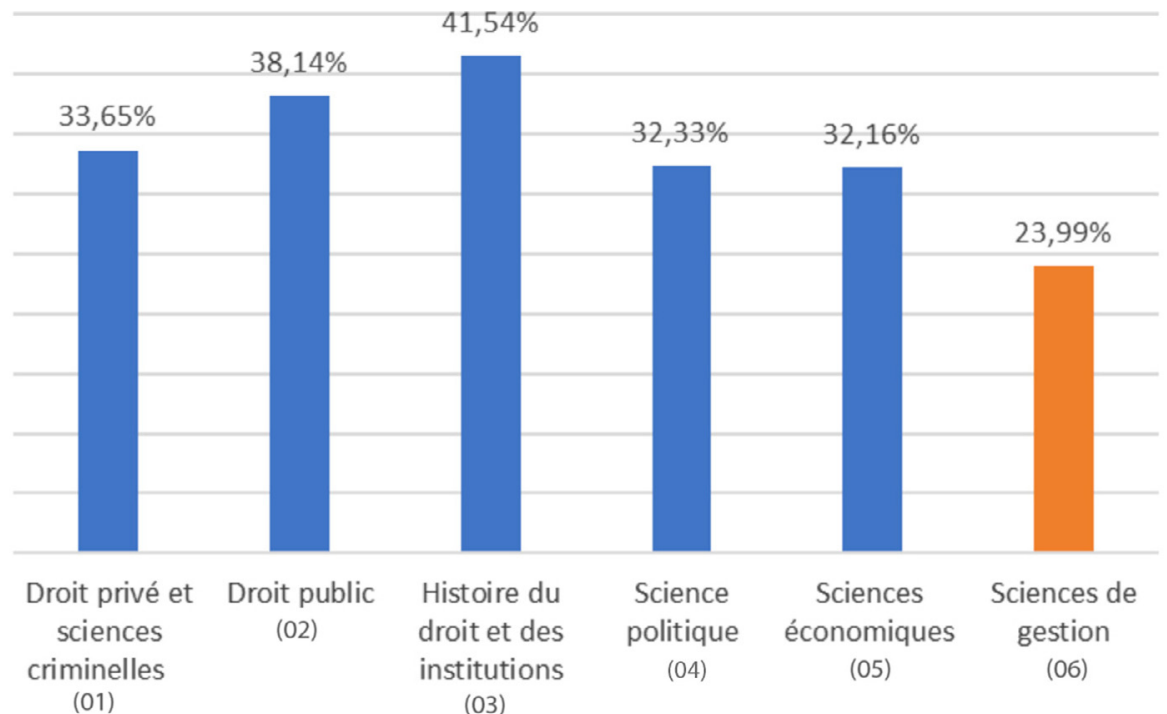

Source : Exploitation des fichiers de gestion de la Direction générale des ressources humaines (RH-SUPINFO ct GESUP2)

pharmacie). En moyenne, en 2019-2020, pour ces disciplines, la proportion de PU parmi les enseignants-chercheurs est de $31,5 \%$. Signalons que certaines sections aux effectifs très petits présentent une proportion de professeurs très élevée qui est un peu artificielle. C'est particulièrement le cas de la section 77 (théologie protestante) qui comporte $4 \mathrm{MCF}$ et $14 \mathrm{PU}$. C'est pourquoi la moyenne pour ces sections a été calculée en reprenant l'ensemble des postes toutes sections confondues (figure 2). En dépit de ces chiffres faibles pour notre section, nous voyons deux lueurs d'espoir. Tout d'abord, regardons l'évolution dans le temps de la part des PU parmi les enseignants-chercheurs. Si aujourd'hui cette part est de 23,99\%, il est à noter qu'elle était encore plus faible par le passé.
Ce chiffre était de $21,17 \%$ en $2010-2011$ et de $21,57 \%$ en 2015-2016. Comme l'illustre la figure 3 , après une période de stabilité relative, nous connaissons une progression de cet indicateur depuis le début de l'expérimentation portant sur le décontingentement.

Ces chiffres laissent penser que l'introduction de la voie du 46-1 et le décontingentement ont permis une légère progression de la part des PU dans les enseignants-chercheurs. Comparons cette évolution à celle connue par la section 05 (sciences économiques) qui elle aussi participait à cette expérimentation (figure 4).

Là encore, il semble que cette expérimentation ait eu un effet positif.

Intéressons-nous maintenant aux disciplines juridiques. Ces dernières n'ayant pas 
Figure 2 - Part des PU au sein des enseignants-chercheurs (sections CNU des grandes disciplines droit, lettres, sciences, pharmacie) (2019-2020)

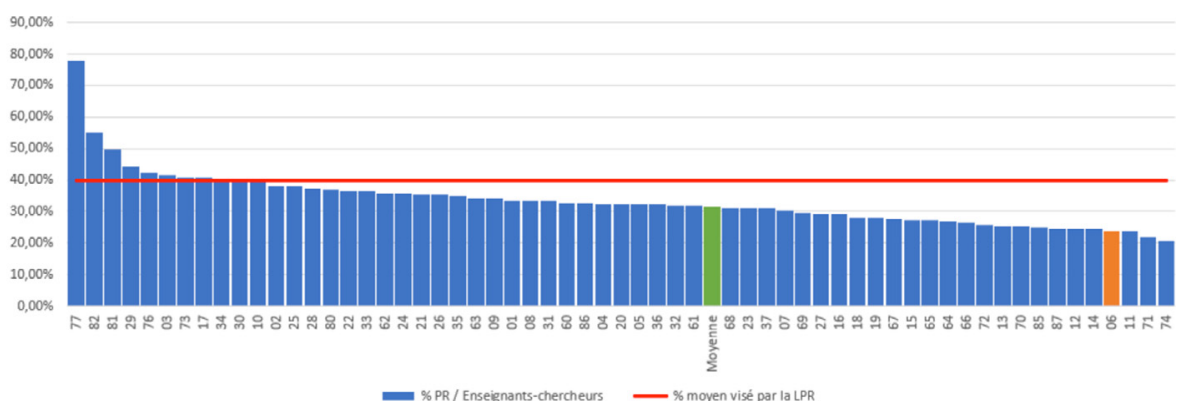

Source : Exploitation des fichiers de gestion de la Direction générale des ressources humaines (RH-SUPINFO et GESUP2)

Figure 3 - Part des PU au sein des enseignants-chercheurs (section 06) (2010-2019)

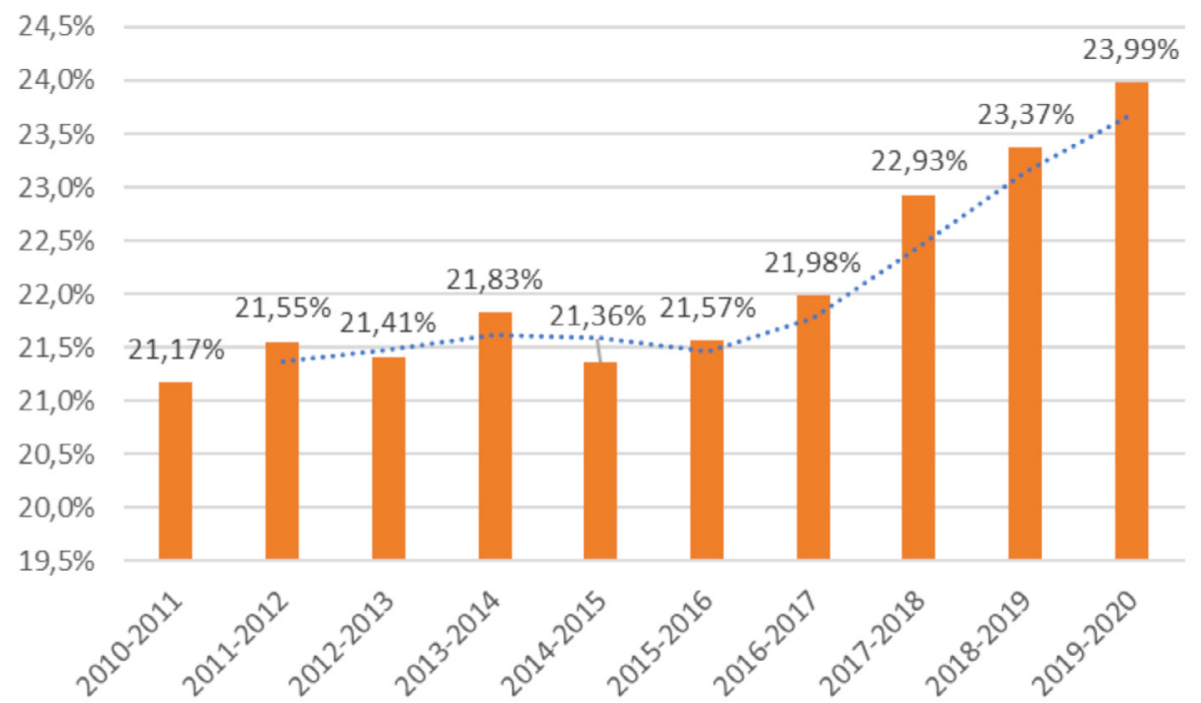

Source : Exploitation des fichiers de gestion de la Direction générale des ressources humaines (RH-SUPINFO ct GESUP2)

participé à cette expérimentation ne connaissent pas d'évolution similaire. Les sections 01 à 03 (figures 5 à 7) connaissent un période de décrue puis de rattrapage.
En ce qui concerne la section 04 (science politique), elle connaît un recul continu (figures 8).

La deuxième lueur d'espoir pourrait se trouver dans les dernières mesures prises par 
Figure 4 - Part des PU au sein des enseignants-chercheurs (section 05) (2010-2019)

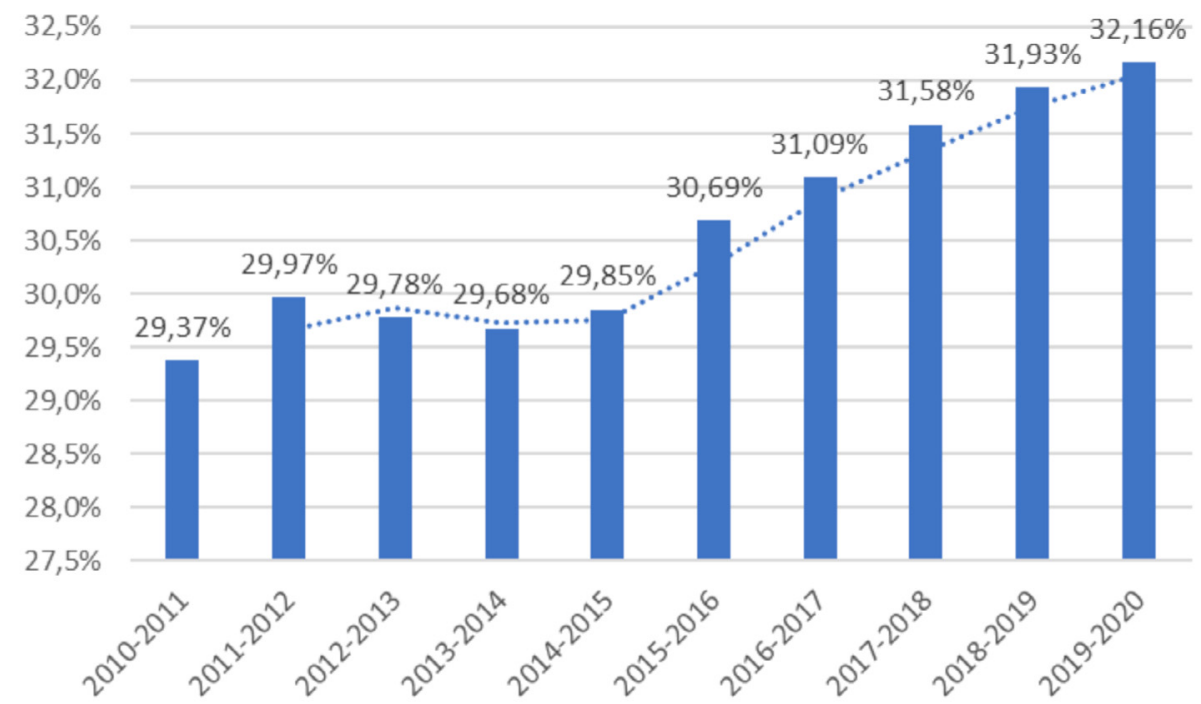

Source : Exploitation des fichiers de gestion de la Direction générale des ressources humaines (RH-SUPINFO et GESUP2)

le gouvernement. En effet, la LPR s'accompagne d'un protocole sur les rémunérations et carrières qui modifie primes et déroulement des carrières des personnels ${ }^{3}$. Ce dernier a été signé en octobre 2020 et fixe notamment l'objectif de constituer un corps de professeurs représentant au moins $40 \%$ du nombre d'enseignants-chercheurs. Pour atteindre cet objectif, il est prévu de permettre à des maîtres de conférences « expérimentés » de bénéficier d'une voie de recrutement réservée pour accéder aux corps des professeurs d'université. Cette nouvelle voie pourrait nous permettre de rattraper une partie de notre retard en ce qui concerne la part des PU parmi les enseignantschercheurs.

Ainsi, même si la part des PU parmi les enseignants-chercheurs est très faible pour la section 06, le décontingentement semble avoir eu un effet positif et les mesures envisagées par le gouvernement pourraient encore améliorer la situation.

\section{III - OÙ EST ALORS LA MENACE ?}

Pour justifier ces menaces, l'article de Paché (2021) évoque le retour d'un possible « lien organique entre processus centralisé (postes mis au concours

3. Loi de programmation de la recherche 2021-2030 : signature d'un accord historique sur les rémunérations et les carrières. (2020, octobre 19). Ministère de l'Enseignement supérieur, de la Recherche et de l'Innovation. https://www. enseignementsup-recherche.gouv.fr/cid154664/loi-de-programmation-de-la-recherche-2021-2030-signature-d-unaccord-historique-sur-les-remunerations-et-les-carrieres.html 
Figure 5 - Part des PU au sein des enseignants-chercheurs (section 01) (2010-2019)

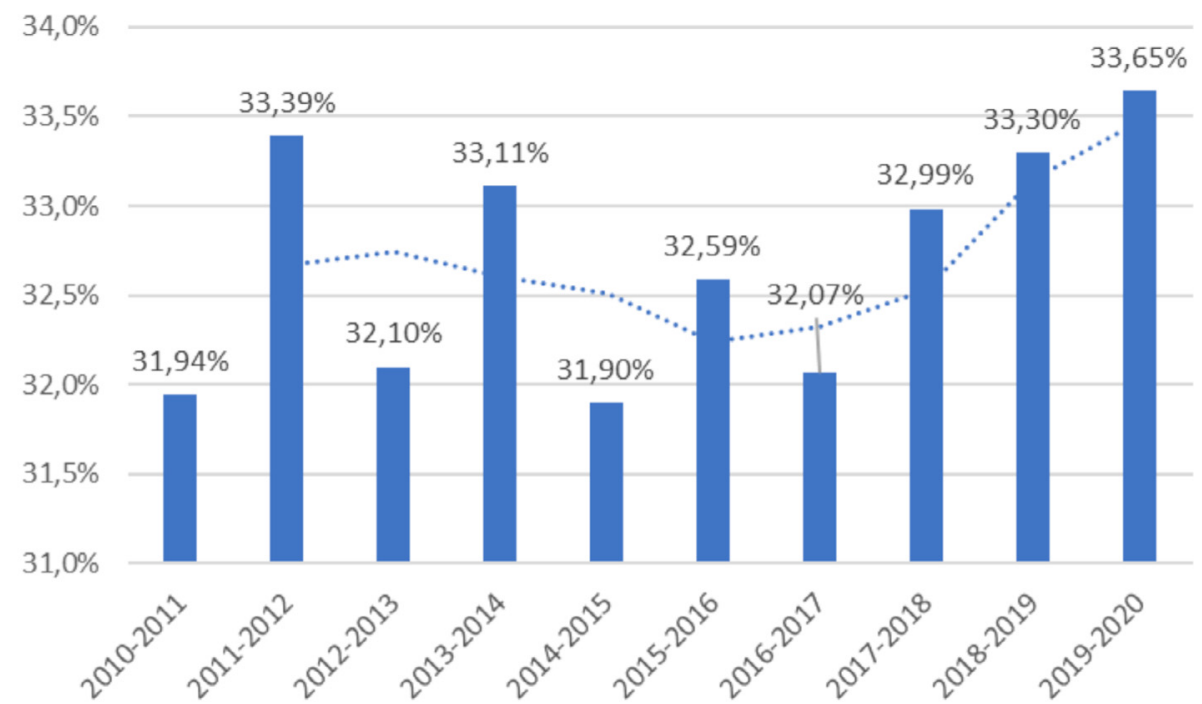

Source : Exploitation des fichiers de gestion de la Direction générale des ressources humaines (RH-SUPINFO et GESUP2)

d'agrégation) et processus décentralisé (postes offerts au recrutement local) ». À travers un détour par le dilemme du prisonnier, l'article présente les menaces qui pèseraient alors sur les recrutements de professeurs en section CNU 06 si les universités ne parvenaient pas à coopérer. Cette option du contingentement semble dans l'immédiat inévitable en raison de l'arrêt de cette expérimentation qui conduit au retour du dispositif existant avant cette expérimentation. Son retour est malgré tout surprenant. En effet, le rapport de l'Hcéres (Cosnard et Dubois, 2019) indiquait que : «Pour autant, un retour au contingentement n'est ni acceptable, ni susceptible de remédier à cette situation. [...] Au demeurant, le contingentement va clairement à l'encontre de la volonté de la quasi-totalité des universités et se traduit en conséquence par une limitation des postes sur chaque voie qui ne correspond ni aux besoins des territoires, ni aux attentes des candidats. »

Cependant, dans une interview donnée à News Tank ${ }^{4}$ par Éric Lamarque (président du CNU 06 de 2011 à 2015) et Aude Deville (présidente du CNU 06 de 2019 à 2023), cette dernière se félicite d'une discussion constructive avec le ministère qui a conduit à la

4. CNU : les sciences de gestion plaident pour une procédure nationale dans le recrutement des PR (22/02/2021). News Tank. https://education.newstank.fr/fr/tour/news/209188/cnu-sciences-gestion-plaident-procedure-nationalerecrutement-pr.html 
Figure 6 - Part des PU au sein des enseignants-chercheurs (section 02) (2010-2019)

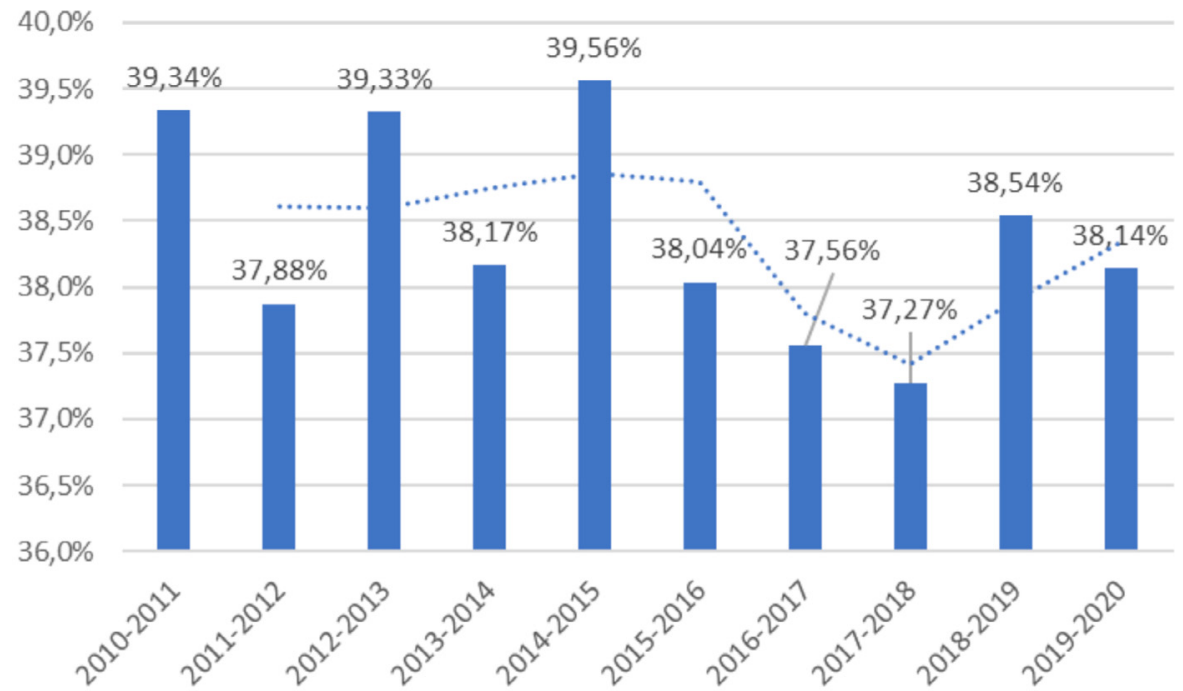

Source : Exploitation des fichiers de gestion de la Direction générale des ressources humaines (RH-SUPINFO et GESUP2)

Figure 7 - Part des PU au sein des enseignants-chercheurs (section 03) (2010-2019)

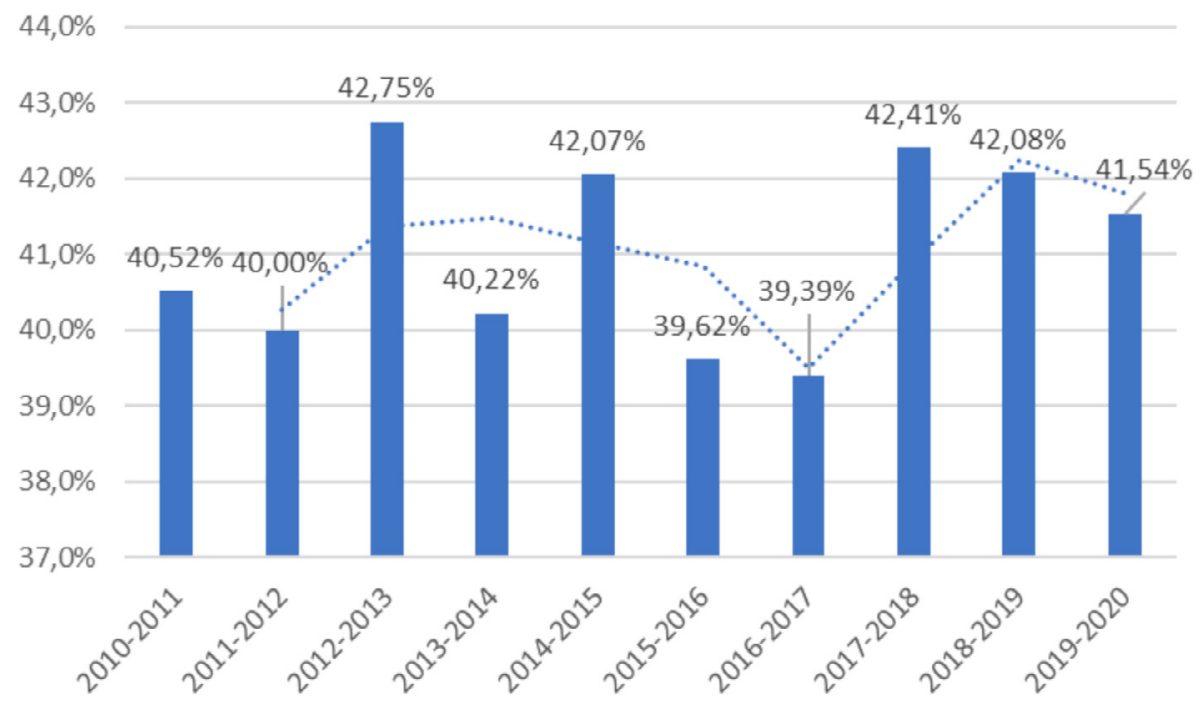

Source : Exploitation des fichiers de gestion de la Direction générale des ressources humaines (RH-SUPINFO et GESUP2) 
Figure 8 - Part des PU au sein des enseignants-chercheurs (section 04) (2010-2019)

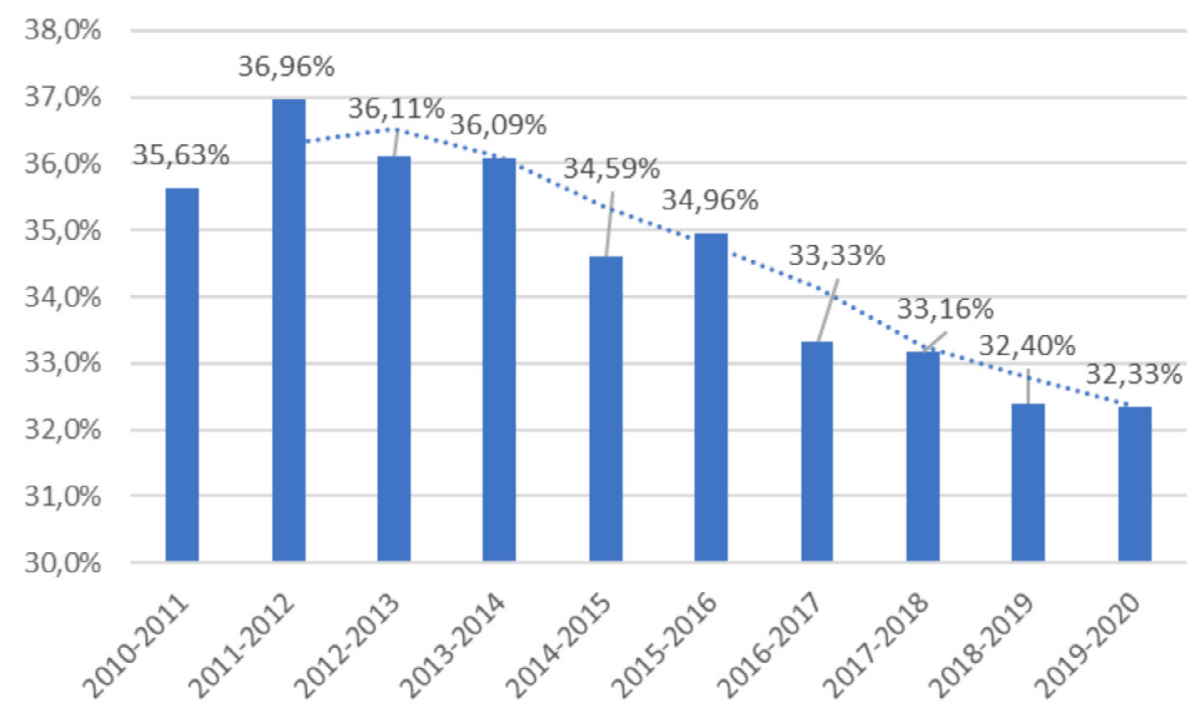

Source : Exploitation des fichiers de gestion de la Direction générale des ressources humaines (RH-SUPINFO et GESUP2)

reprise d'une proposition de contingentement : « un contingentement un pour deux (pour un poste d'agrégé ouvert, deux postes en 46-1 potentiels pourront être ouverts sur deux ans avec un décalage d'un an) ».

Ce retour du contingentement n'est donc, dans l'immédiat, plus une option et il est porté par l'opposition affichée depuis longtemps par certains représentants du CNU 06 à l'égard du décontingentement. Ainsi, Éric Lamarque, indique dans cette interview à News Tank : « [...] j'étais opposé à ce décontingentement. [...] Bref, je considère cette expérimentation comme largement négative et n'a provoqué aucun bénéfice globalement. Je salue donc le rétablissement $\mathrm{du}$ concours et d'un nombre minimum de postes à pourvoir par cette voie. ». Aude Deville, présidente actuelle du CNU, précise que : «En termes de $\mathrm{RH}$, pour la discipline le décontingentement a été en la défaveur des collègues. "

Cette position s'appuie également sur le rapport d'évaluation de l'Hcéres sur cette expérimentation (Cosnard et Dubois, 2019). Selon ce rapport, les conséquences du décontingentement auraient été principalement les suivantes :

- une diminution du nombre de postes mis au concours d'agrégation (4 en 2019) et aucune proposition d'emploi de la part des établissements pour le concours ouvert en 2020 au titre de l'année 2021 ; 
- un âge moyen d'accès aux postes de PU plus élevé par la voie du 46-1 que par l'agrégation ;

- un taux d'endorecrutement ${ }^{5}$ élevé par la voie du 46-1. Il passe de $58 \%$ en 2015 2016 à $70 \%$ sur la période $2017-2018^{6}$.

Nous ne reviendrons pas sur la baisse du nombre de postes mis au concours. Il nous semble préférable de nous attacher au nombre global de postes disponibles malgré l'intérêt intellectuel du concours d'agrégation et sa capacité à offrir des carrières rapides à certains lauréats. Globalement, le nombre de PU augmente et c'est de l'intérêt des institutions qui nous accueillent et de la section CNU 06 dans son ensemble (figure 9).

En ce qui concerne l'âge moyen des PU, le rapport Hcéres souligne que la différence entre les PU issus de l'agrégation et ceux de la voie 46-1 n'est pas si élevée que prévu. Notons également que le nombre de lauréats $\mathrm{du}$ concours d'agrégation sur la période 2015-2019 est de 28 alors que, dans le même temps, le nombre de PU recrutés par la voie du 46-1 était de 131 (tableau 1). Le calcul d'un âge moyen pour les lauréats de l'agrégation peut s'avérer un peu aléatoire avec des effectifs faibles ${ }^{7}$. On peut aussi penser que les candidats plus âgés, qui n'auraient pas eu d'autre choix avant 2015 que d'emprunter la voie de l'agrégation, se sont portés sur la voie du 46-1 car ils disposaient d'un dossier cohérent avec cette voie, alors que les plus jeunes, avec un dossier moins étoffé, ont privilégié la voie de l'agrégation. L'introduction de la voie du 46-1 a probablement eu un effet « déstockage » de maîtres de conférences plus âgés qui y ont vu une opportunité.

Si l'on examine les chiffres de l'endorecrutement, l'année 2017 semble « remarquable » avec un taux de 68,75\% (tableau 2). Depuis, le chiffre baisse et nous étions en 2019 à 52,38 \% ce qui est un peu en deçà du chiffre calculé pour l'ensemble des sections. Il était inévitable que l'endorecrutement des PU augmente avec l'introduction du 46-1 et le décontingentement. En effet, avec l'agrégation externe, l'établissement d'affectation des lauréats résulte de leur classement au concours. Être recruté par son établissement d'origine est donc très aléatoire. Ce faible endorecrutement par la voie de l'agrégation externe masque également les retours dans l'université d'origine après 3 ans d'un parachutage pas toujours heureux à jouer les « turbo-professeurs » ou les « professeurs TGV ». Notons également que les chiffres toutes sections confondues montrent une certaine stabilité dans le temps. Même si l'endorecrutement est un risque, on peut probablement encore le réduire par différentes mesures. Par exemple, en contraignant ou en incitant les établissements à recruter en extérieur au moins un poste sur deux.

Au-delà de ces trois éléments mis en avant dans le rapport de l'Hcéres, on peut aussi y lire que « la proportion de femmes parmi les recrutés sur un poste 46,1 est de 6 points

5. L'endorecrutement des PU désigne le recrutement de PU dans l'établissement où ils étaient MCF.

6. Nous ne sommes pas parvenus à reconstituer les chiffres de l'endorecrutement proposés dans le rapport de l'Hcéres. En effet, les données que nous avons trouvées pour la campagne de recrutement de 2015 ne concernent que la campagne synchronisée et pas la campagne au fil de l'eau contrairement aux données disponibles pour les années suivantes. Par ailleurs, il n'est pas clairement dit si ce calcul exclut ou pas les postes proposés à la mutation. Signalons également que le calcul semble être réalisé sur 2 années (2015-2016 et 2017-2018) mais sans que nous en ayons la certitude.

7. Le rapport Hcéres semble d'ailleurs calculer cet âge moyen pour 2015-2016 et 2017-2018. 
Figure 9 - Évolution du nombre de PU de 2010-2011 à 2019-2020 (section 06)

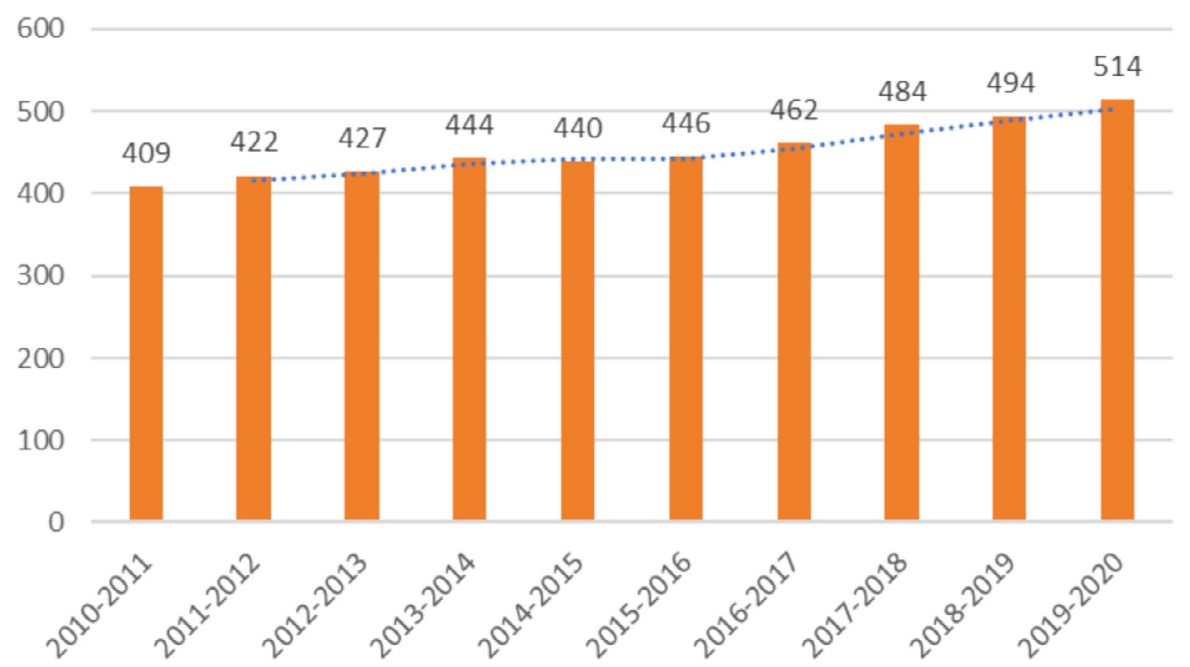

Source : Exploitation des fichiers de gestion de la Direction générale des ressources humaines (RH-SUPINFO et GESUP2)

plus élevée que parmi les agrégés des trois derniers concours : $58,3 \%$ contre $52,1 \%$. ». En ce qui concerne le ratio PU/ $\mathrm{MCF}$, le Hcéres considère qu'il dispose, au moment de l'écriture du rapport, d'une série de données trop courte pour pouvoir vérifier l'hypothèse d'un effet du 46-1 et du décontingentement. Les données que nous fournissons aujourd'hui semblent plutôt aller dans le sens d'un effet positif (figure 3). À l'extérieur de la section CNU 06, le contingentement ne semble pas plébiscité. Ainsi, le Hcéres, dans son évaluation du dispositif de décontingentement des professeurs en sciences économiques (Cosnard et Cartapanis, 2019), a recommandé l'abandon définitif du contingentement. Par ailleurs, dans un communiqué, la CPU (Conférence des présidents d'université) a regretté pour les sciences de gestion que « le rapport du Hcéres ne préconise pas d'aller jusqu'au bout de la démarche de décontingentement, elle espère que la reconduction de l'expérimentation permettra d'y arriver. ${ }^{8}$. Enfin, signalons que le rapport Sawicki (2017) préconisait que la section CNU 04 - science politique, qui ne participait pas à cette expérimentation, adopte à titre expérimental

8. Recrutement des professeurs et décontingentements. (2019, septembre 30). CPU - Conférence des présidents d'université. http://www.cpu.fr/actualite/recrutement-des-professeurs-et-decontingentements/ 
Tableau 2 - Taux d'endorecrutement

\begin{tabular}{|l|c|c|c|c|}
\hline $\begin{array}{l}\text { Taux d'endorecrutement par la voie du 46-1 } \\
\text { (y compris 46-5, hors mutation et détachement, } \\
\text { hors 46-3, hors agrégation) }\end{array}$ & $\mathbf{2 0 1 6}$ & $\mathbf{2 0 1 7}$ & $\mathbf{2 0 1 8}$ & $\mathbf{2 0 1 9}$ \\
\hline Section CNU 06 & $56,25 \%$ & $68,75 \%$ & $59,09 \%$ & $52,38 \%$ \\
\hline Toutes sections confondues & $56,22 \%$ & $57,44 \%$ & $58,66 \%$ & $53,38 \%$ \\
\hline
\end{tabular}

ce dispositif face aux difficultés de recrutement de professeurs en science politique ${ }^{9}$.

En définitive, l'article de Paché (2021) souligne parfaitement les menaces que fait peser le contingentement sur notre section, mais omet de s'interroger sur son intérêt. Pourquoi arrêter le décontingentement tel qu'il existait à titre expérimental ? C'est probablement plutôt dans cet arrêt et le retour du contingentement que se trouve la menace qui pèse sur le corps des PU.

Certes, le décontingentement favorise probablement l'endorecrutement, mais ce phénomène devrait pouvoir être contenu comme il l'est pour les autres sections CNU. À terme, il pourrait aussi faire peser un risque sur la possibilité de maintenir le concours d'agrégation et ainsi priver de jeunes enseignantschercheurs d'une carrière accélérée. Cependant, le nouveau dispositif de chaires de professeurs juniors aura aussi cette fonction tout en permettant l'émergence de profils transversaux. Comment parviendrons-nous à nous emparer de ce nouveau dispositif tout en imposant un nombre de postes à l'agrégation à travers le contingentement ?

Le maintien du décontingentement pourrait présenter des désagréments. Mais, comme nous l'avons vu, globalement, sa mise en place et l'introduction du 46-1 ont eu un effet positif pour notre section en améliorant le ratio PU/enseignants-chercheurs et en facilitant l'accès des femmes au corps des PU. Dès lors, ne devons-nous pas en accepter les inconvénients ?

Avec le dilemme du prisonnier, Paché (2021) souligne les dangers qu'il y a quand les acteurs cherchent à privilégier leur intérêt individuel. Cependant, Smith (2014 [1759]), dans son ouvrage sur la «théorie des sentiments moraux » montre aussi toute l'importance $\mathrm{du}$ concept de sympathie et le rôle que doit jouer notre « spectateur impartial » dans les décisions que nous prenons. C'est notre capacité à comprendre l'autre, à nous décentrer qui nous permettra de maîtriser notre égoïsme naturel afin de ne pas nuire aux autres. Il en va des universités, qui devront s'efforcer de coopérer dans le cas d'un retour définitif du contingentement, comme des différents acteurs qui pèseront ou non pour son retour. Comme le souhaitait la CPU, la poursuite de cette expérimentation nous permettrait, pour le moins, de disposer d'un peu plus de recul sur un décontingentement qui semble porter ses fruits.

9. Frédéric Sawicki préconisait dans son rapport le décontingentement expérimental pour quatre ans. Cette recommandation fut appuyée par une pétition ainsi que par la section 04 du CNU qui avait pris position début 2017 en faveur d'un décontingentement et d'un maintien du concours d'agrégation sous des formes modifiées. Il en était de même pour la CP-CNU pour les sections du groupe 1 . 


\section{BIBLIOGRAPHIE}

Cosnard M. et Dubois P.-L. (dir.) (2019). Évaluation du dispositif expérimental de recrutement des professeurs des universités en sciences de gestion, Haut Conseil de l'évaluation de la recherche et de l'enseignement supérieur, Paris.

Cosnard M. et Cartapanis A. (dir.) (2019). Évaluation du dispositif expérimental de recrutement des professeurs des universités en sciences économiques, Haut Conseil de l'évaluation de la recherche et de l'enseignement supérieur, Paris.

Musselin C. (2020). «Les paradoxes de la LPPR ou pourquoi l'annonce de moyens supplémentaires est-elle source de résistances ? », Revue française de pédagogie, vol. 46, $\mathrm{n}^{\mathrm{o}} 207$, p. 41-48.

Paché G. (2021). « Menaces sur le corps des professeurs des universités en sciences de gestion et du management ? », Revue française de gestion, vol. 47, $\mathrm{n}^{\circ} 294$, p. 41-51.

Sawicki F. (2017). Pour une réforme des modalités d'accès au corps des professeurs en science politique, Secrétariat d'État chargé de l'Enseignement supérieur et de la Recherche, Paris.

Smith A. (1759/2014). Théorie des sentiments moraux, Presses Universitaires de France - PUF.

Stiegler B. et Pébarthe C. (2020). « Gouverner, c'est détruire. Ou comment la LPPR détruit la recherche en la finançant », Revue française de pédagogie, $\mathrm{n}^{\mathrm{o}} 207$, p. 69-77. 